\title{
Torsión de ovario por cistoadenoma en una recién nacida
}

\section{Ovarian torsion due to cystadenoma in a newborn}

\author{
Alfredo Chama-Naranjo, * Cuauhtémoc Borja-Villa, ${ }^{\ddagger}$ Venus Medina-Illueca, ${ }^{\S}$ Julieta Robles-Castro, ${ }^{\natural}$ \\ Francisco Javier Luna-Aguilar, J Jorge Farell-Rivas,** María Irene Rivera-Salgado \\ * Servicio de Cirugía General; ‡ Residente de Cirugía General; § Residente de Anatomía Patológica; \\ " Oncóloga pediatra; " Cirujano pediatra; ** Departamento de Cirugía General; ${ }^{\#}$ Servicio de \\ Patología. Hospital Central Sur de Alta Especialidad, PEMEX, Ciudad de México.
}

\section{RESUMEN}

Objetivo: Describir el caso de una recién nacida con tumor de ovario congénito. Presentación de caso: El tumor se detectó por ultrasonido fetal, y a los dos días de vida se realiza escisión quirúrgica de tumor quístico de ovario derecho de forma exitosa, sin complicaciones. El estudio histopatológico reportó un cistoadenoma seroso. Conclusión: Los tumores de ovario congénitos son infrecuentes. El abordaje quirúrgico permite establecer el diagnóstico y dar un tratamiento definitivo.

Palabras clave: Cistoadenoma seroso, tumores neonatales, tumor ovárico, alfafetoproteína.

\section{INTRODUCCIÓN}

Los tumores congénitos son infrecuentes, se calculan en 0.5-2\% y se diferencian en tipo, incidencia y evolución a los del adulto. ${ }^{1}$ Los tumores de origen ovárico en pediatría ocurren aproximadamente en 2.6/100,000 casos por año, entre 10 y $20 \%$ son malignos y representan $3 \%$ de los cánceres en niñas menores de 15 años. ${ }^{2} \mathrm{El}$ diagnóstico debe considerarse ante la presencia de dolor abdominal y un tumor palpable. . $^{3,4}$

\begin{abstract}
Objective: To describe the case of a newborn with a congenital ovarian tumor. Case presentation: The tumor was detected by fetal ultrasound. At two days of life, a surgical excision of the right ovarian cystic tumor was performed without complications. Conclusion: Congenital ovarian tumors are rare. The surgical approach allows establishing the diagnosis and giving a definitive treatment.
\end{abstract}

Keywords: Serous cystadenoma, neonatal tumors, ovarian tumor, alpha-fetoprotein.

Los tumores de ovario en la edad pediátrica son generalmente benignos. Pueden ser simples (quísticos) o complejos (contienen áreas sólidas y quísticas). Es muy probable que un tumor de ovario quístico persistente, se trate de cistoadenoma mucinoso o seroso. ${ }^{5}$ La Organización Mundial de la Salud (OMS) clasifica a los tumores de ovario según su histología y su comportamiento benigno o maligno. ${ }^{2}$ La mayoría de los tumores de ovario en pediatría son de origen germinal, en comparación con los tumores epiteliales en mujeres. ${ }^{6}$

Correspondencia: Alfredo Chama-Naranjo, alfredchamaa@gmail.com

Citar como: Chama-Naranjo A, Borja-Villa C, Medina-Illueca V, Robles-Castro J, Luna-Aguilar FJ, Farell-Rivas J et al. Torsión de ovario por cistoadenoma en una recién nacida. Rev Mex Pediatr. 2021; 88(1): 26-30. https://dx.doi.org/10.35366/99416 


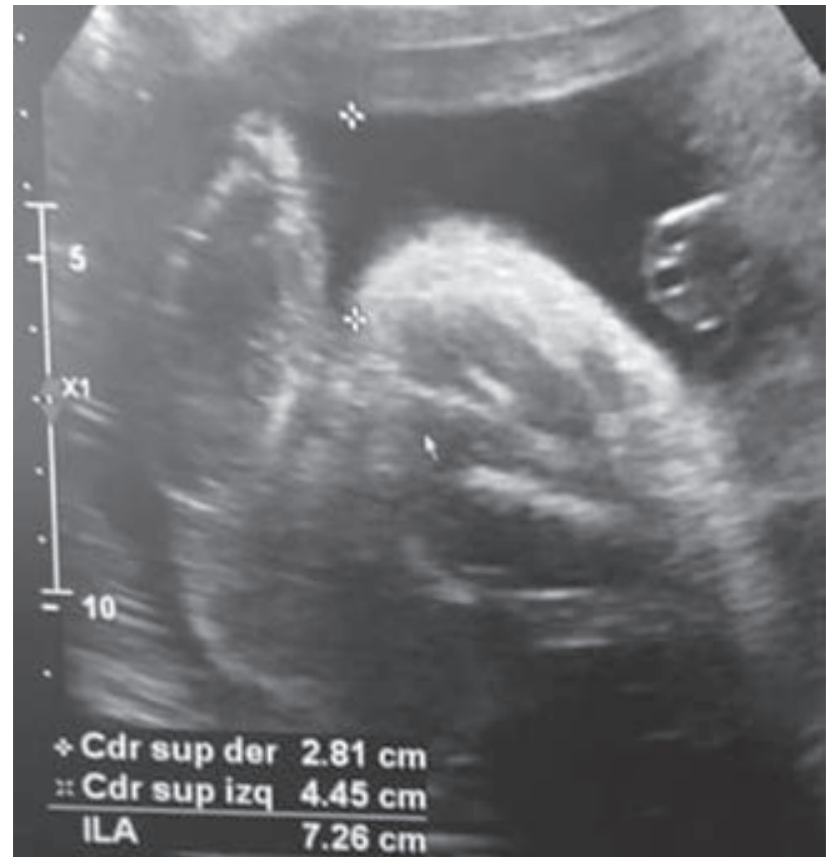

Figura 1: Ultrasonido prenatal 38 semanas de gestación.

Los más frecuentes corresponden a quistes de ovario no neoplásico y son de origen folicular $(50 \%) .{ }^{7} \mathrm{El}$ cistoadenoma, teratoma quístico y el tumor de células granulosas son extremadamente raros. ${ }^{4}$ Cuando se determina que hay incremento en el tamaño del ovario, ya sea quístico o sólido, debe evaluarse a fin de excluir malignidad. ${ }^{8}$
El objetivo de este artículo es describir el caso de una recién nacida $(\mathrm{RN})$ con cistoadenoma seroso detectado de forma intrauterina, dependiente de ovario derecho, puesto que al revisar la literatura no se encontraron casos similares.

\section{PRESENTACIÓN DEL CASO}

$\mathrm{RN}$ femenino, producto de segunda gesta con control prenatal desde el primer trimestre, con 15 consultas. A partir de la semana 28 se detectó diabetes gestacional, siendo el tratamiento a base de restricción de calorías. Además, en la semana 29 se detectó candidiasis vaginal, que se trató con isoconazol, en dosis única. En la semana 35 presentó cervico-vaginitis, recibiendo fluocinolona, nistatina y metronidazol.

Durante el embarazo se realizaron tres ultrasonidos obstétricos, y se detectó quiste abdominal en la semana 32 en la fosa iliaca derecha, con dimensiones de $18 \times 17 \mathrm{~mm}$, que no condiciona compresión o efecto de masa. Para la semana 38, en un nuevo ultrasonido, se determinó que el quiste medía $43 \times 35 \times 38 \mathrm{~mm}$, con un volumen de $30.8 \mathrm{~cm}^{3}$ (Figura 1).

El parto fue eutócico en la semana 38 de gestación, obteniendo producto de 2.7 kilogramos y talla de $50 \mathrm{~cm}$ con perímetro abdominal de 31 centímetros. En la exploración física se identificó un tumor palpable en cuadrantes inferiores del abdomen. Al segundo día de vida es referida a nuestra institución, donde se realizó un ultrasonido, el cual reportó, a nivel de flanco derecho, imagen heterogénea, formando un

Figura 2:

Tumor heterogéneo, formando un nivel con pared ecogénica sin señal al Doppler color, con dimensiones de 43 $\times 31 \times 38 \mathrm{~mm}$ con un volumen de $27.7 \mathrm{~cm}^{3}$.
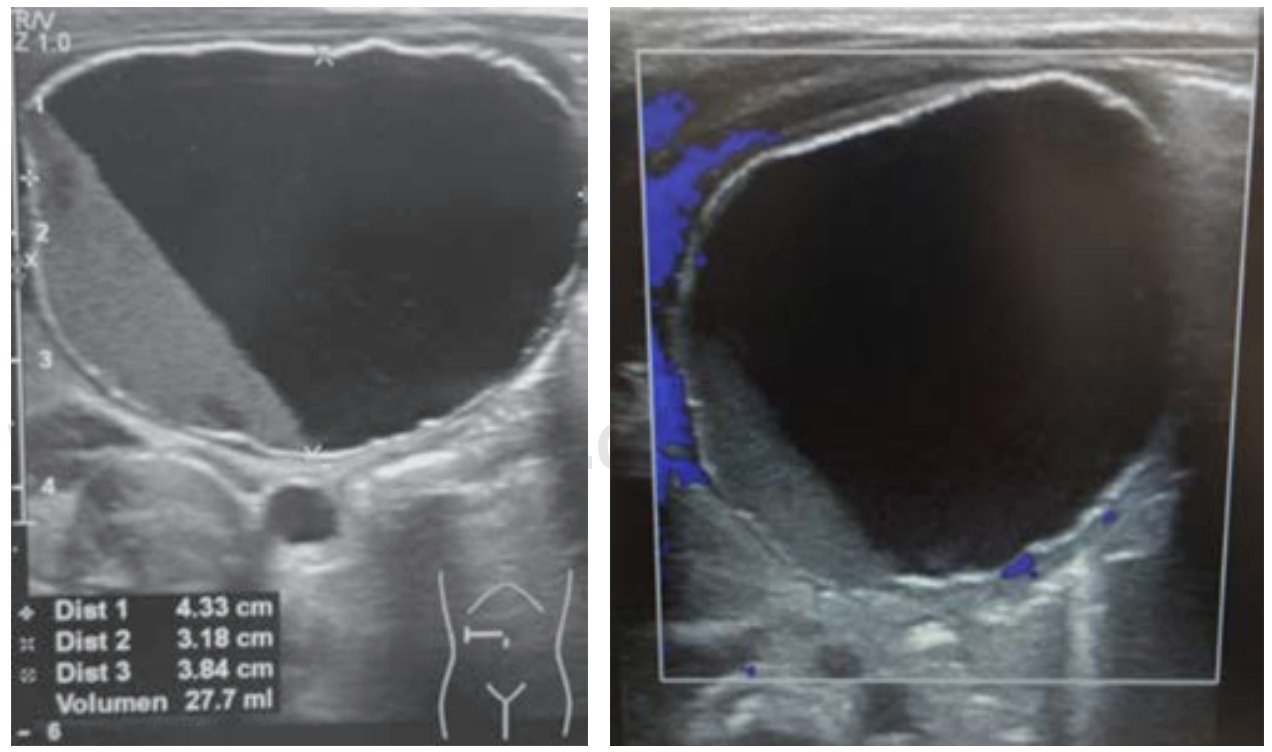
nivel líquido, ovoideo, circunscrita, con pared ecogénica sin señal al Doppler color, con dimensiones de $43 \times 31 \times 38 \mathrm{~mm}$, con un volumen aproximado de $27.7 \mathrm{~cm}^{3}$. Esta masa no condicionaba desplazamiento de estructuras abdominales o datos de obstrucción. La impresión diagnóstica fue de torsión de ovario (Figura 2).

Ese mismo día se procedió a realizar laparotomía exploradora, en la que se encontró un tumor quístico de ovario derecho de $40 \times 40 \mathrm{~mm}$, con adherencias a apéndice cecal e intestino delgado. Se realizó apendicetomía incidental por adherencias del tumor más salpingooforectomía derecha (Figuras 3 y 4). No se encontró invasión del tumor hacia las estructuras.

El reporte histopatológico reportó: tumor de ovario derecho compatible con cistadenoma seroso de ovario calcificado, con extensa congestión vascular, edema y necrosis isquémica, apéndice cecal sin alteraciones adherido a la pared del tumor quístico. Se consideró que los cambios isquémicos y las calcificaciones eran compatibles con torsión prenatal del tumor de larga evolución. La salpinge se observó con extensa congestión vascular (Figura 5).

La RN permaneció hospitalizada durante cinco días, con buena evolución, por lo que egresó por mejoría. Acudió a consulta un mes posterior a cirugía; el reporte de alfafetoproteína (AFP) presentó dilución 1:100 = $79,257 \mathrm{ng} / \mathrm{mL}$ (normal para la edad). El control al mes siguiente de AFP fue de $1,263.54 \mathrm{ng} / \mathrm{mL}$, por lo que

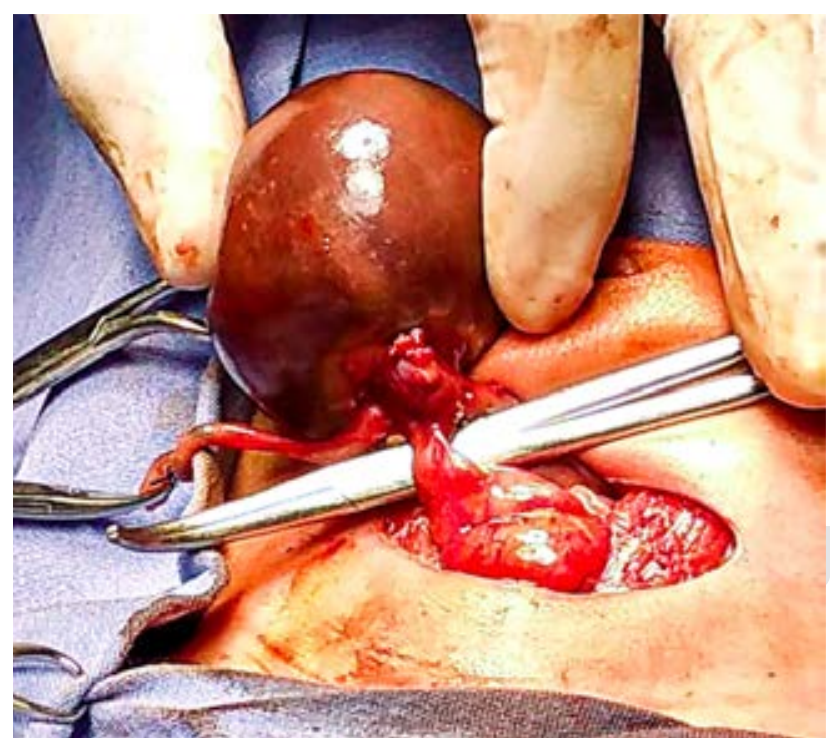

Figura 3: Torsión del tumor adherido a salpinge derecha y apéndice cecal.

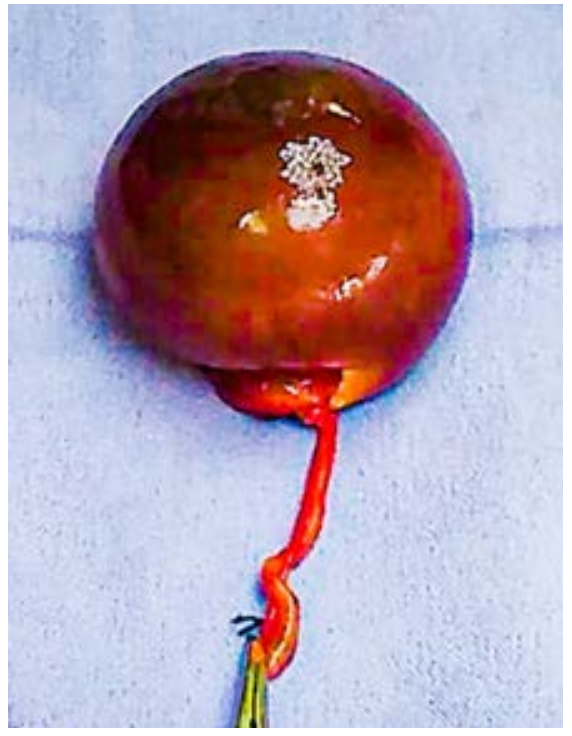

Figura 4: Cistoadenoma seroso con apéndice cecal.

se decidió su alta del servicio de Cirugía y Oncología Pediátrica.

\section{DISCUSIÓN}

En el 2015, Hermans y cols. señalan el primer caso de una $\mathrm{RN}$ con un quiste de ovario roto que fue sometida a laparotomía. ${ }^{4}$ Valenti y colaboradores detectaron quistes de ovario como hallazgos prenatales, y se creía que eran raros. ${ }^{5}$ Con las nuevas tecnologías de imagen, los quistes ováricos fetales se han detectado con mayor frecuencia. ${ }^{9}$

La mayoría de los tumores de ovario en pacientes pediátricos son benignos. Cribb y su grupo ${ }^{10}$ observaron que las lesiones de ovario más frecuentes incluían quistes ováricos simples, quistes paraováricos y quistes hemorrágicos. Los quistes pueden ser simples o complejos y comúnmente son unilaterales. Se pueden presentar como complicaciones intrauterinas: torsión y ruptura, y las opciones de tratamiento abarcan desde tratamiento conservador hasta la aspiración prenatal, parto temprano y cirugía neonatal. ${ }^{11}$ En nuestra paciente se encontró una torsión del tumor, por lo que consideramos la cirugía como tratamiento.

Nussbaum clasificó los quistes de ovario fetales en dos grupos de acuerdo con los hallazgos de ultrasonido: simples y complicados. Las características de los quistes no complicados son: anecógenas, redondos, $>20 \mathrm{~mm}$ de diámetro, uniloculares, intrapélvicos o 

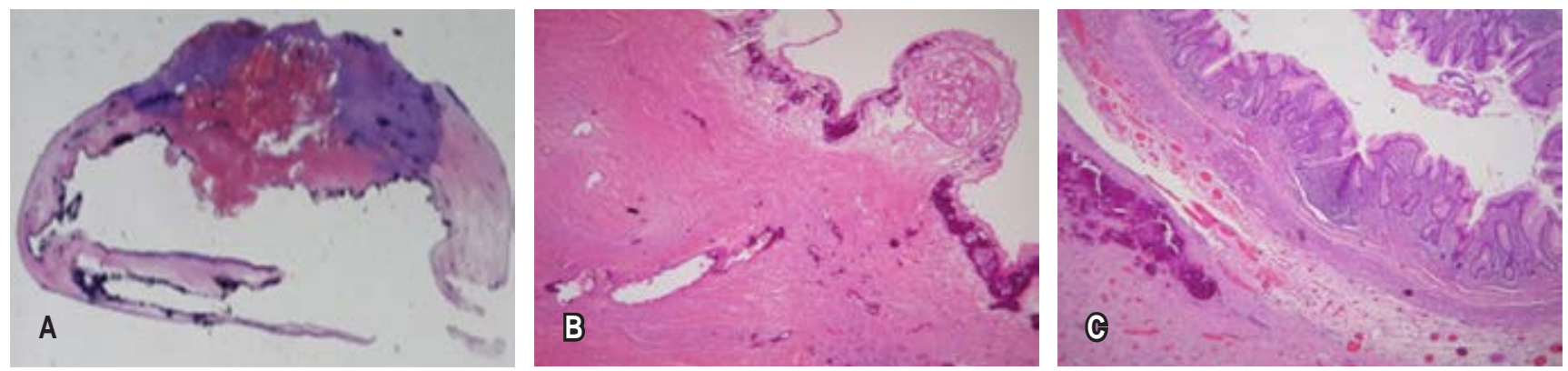

Figura 5: A) Aspecto panorámico del corte completo de la lesión quística, con borde luminal calcificado y zona de esclerosis con material hemático adherido, similar al que contenía todo el quiste. B) Corte histológico H\&E de la pared quística con extensa calcificación del borde luminal asociada a la torsión del tumor, sin epitelio residual de revestimiento. C) H\&E, pared del quiste con calcificación y extensa congestión vascular entre la cápsula del quiste y la serosa del apéndice cecal, esta última sin alteraciones histológicas.

intraabdominales, unilaterales y raramente bilaterales; además son de pared delgada y pueden moverse con las posiciones de la madre. Por su parte, los quistes complicados son heterogéneos, con componentes hiperecogénicos, de paredes gruesas, contienen material flotante, con tabiques intraquísticos y móviles después de una torsión anexial. Cuando hay ausencia de flujo Doppler de color sugiere altamente la torsión. ${ }^{12}$ El ultrasonido en nuestra paciente presentó imagen heterogénea con material flotante y sin señal al Doppler color, por lo que se sospechó torsión del tumor.

Las neoplasias neonatales del ovario son extremadamente raras, $60 \%$ se originan de las células germinales y generan grandes dimensiones, complejas y sólidas. ${ }^{13}$ Los tumores de ovario pueden provenir de tres líneas, según la clasificación histológica de la OMS: 1) epitelio celómico superficial, 2) células germinales, y 3) estroma ovárico. Nuestro caso corresponde al primer grupo en el que existen tres tipos principales: serosos, mucinosos y endometrioides. Los elementos de estos tumores pueden estar compuestos de áreas quísticas (cistoadenomas), áreas quísticas y fibrosas (cistoadenofibromas) y áreas predominantemente fibrosas (adenofibromas). ${ }^{14}$

El reporte de histopatología de nuestra paciente correspondió a cistoadenoma seroso. El comportamiento de los tumores serosos depende del grado de diferenciación y extensión, lo cual determina el pronóstico y el tratamiento. ${ }^{15} \mathrm{El}$ tratamiento con la preservación del ovario (cistectomía) es una alternativa; sin embargo, Chu SM y equipo ${ }^{16}$ observaron que el procedimiento más realizado es la salpingooforectomía. En nuestra paciente se decidió realizar resección de las estructuras comprometidas, dado que se detectaron adherencias del ovario derecho, la tuba ovárica y el apéndice cecal.

La evaluación de los tumores de células germinales puede ser monitorizado a través de marcadores tumorales como la AFP y gonadotropina coriónica fracción beta (hCG- $\beta$ ). La AFP es un marcador confiable para la detección de recurrencia de la enfermedad e iniciar el manejo adecuado. ${ }^{17}$

\section{REFERENCIAS}

1. Albert A, Cruz O, Montaner A, Vela A, Badosa J, Castañón $M$ et al. Tumores sólidos congénitos. Revisión de 13 años. Cir Pediatr. 2004; 17: 133-136.

2. Pérouxa E, Franchi-Abella S, Sainte-Croix D, Canale S, Gauthier F, Martelli H et al. Ovarian tumors in children and adolescents: a series of 41 cases. Diagnostic and Interventional Imaging. 2015; 96(3): 273-282. doi: 10-1016/j. dii.2014.07.001.

3. Heo SH, Kim JW, Shin SS, Jeong SI, Lim HS, Choi YD et al. Review of ovarian tumors in children and adolescents: radiologicpathologic correlation. RadioGraphics. 2014; 34(7): 2039-2056. doi: $10.1148 / \mathrm{rg} .347130144$.

4. Hermans AJ, Kluivers KB, Wijnen MH, Bulten J, Massuger LF, Coppus SF. Diagnosis and treatment of adnexal masses in children and adolescents. Obstet Gynecol. 2015; 125: 611. doi: 10.1097/ AOG.0000000000000665.

5. Valenti C, Kassner EG, Yermakow V, Cromb E. Antenatal diagnosis of a fetal ovarian cyst. Am J Obstet Gynecol. 1975; 15: 216-219.

6. Bhattacharyya NK, De A, Bera P, Sristidhar M, Chakraborty $S$, Bandopadhyay R. Ovarian tumors in pediatric age group: a clinicopathologic study of 10 years' cases in West Bengal, India. Indian J Med Paediatr Oncol. 2010; 31(2): 54-57. doi: 10.4103/0971-5851.71656.

7. Papic JC, Finnell SM, Slaven JE, Billmire DF, Rescorla FJ, Leys $\mathrm{CM}$. Predictors of ovarian malignancy in children: overcoming clinical barriers of ovarian preservation. J Pediatr Surg. 2014; 49 (1): 144-147. doi: 10.1016/j.jpedsurg.2013.09.068. 
8. Lee JH, Tang JR, Wu MZ, Ni YH, Hsu WM. Ovarian cyst with torsion presenting as a wandering mass in a newborn. Acta Paediatr Taiwan. 2003; 44 (5): 310-312.

9. Yilmaz Y, Demirel G, Ulu HO, Celik IH, Suna-Oguz S, Erdeve O et al. Four neonates with giant ovarian cysts: difficulties in diagnosis and decision making process. J Matern Fetal Neonatal Med. 2012; 25 (8): 1508-1510.

10. Cribb B, Vishwanath N, Upadhyay V. Paediatric ovarian lesions the experience at Starship Children's Hospital, New Zealand. New Zeal N. Z Med J. 2014; 127(1395): 41-51.

11. Ibrahim H, Lewis D, Harrison GK, Tice H, Sangster G. Congenital ovarian cyst. J Perinatol. 2007; 27: 523-526.

12. Nussbaum AR, Sanders RC, Hartman DS. Neonatal ovarian cysts: sonographic-pathologic correlation. Radiology. 1988; 168(3): 817821. doi: 10.1148/radiology.168.3.3043551.

13. Alsuhaibani M, Al Harbi M, Al-Lababidi NH, Almuzaini AS, Alsulaiman A, Aljadaan SA. Ovarian lesions among pediatric patients: A tertiary center experience (1997-2016). Int J Pediatr Adolesc Med. 2017; 4(4): 129-132. doi: 10.1016/j. ijpam.2017.08.00.

14. Cowan RA, Haber EN, Faucz FR, Stratakis CA, Gomez-Lobo V. Mucinous cystadenoma in children and adolescents. $J$ Pediatr Adolesc Gynecol. 2017; 30(4): 495-498. doi: 10.1016/j. jpag.2017.02.001.

15. Islas LP, Cardiel LE, Ortiz MaR, Chavez C. Cistoadenoma gigante de ovario en una recién nacida. Rev Mex Pediatr. 2003; 70(1): 19-22.

16. Chu SM, Chao HC, Lai JY, Chen JC, Yung CP et al. Ovarian tumors in the pediatric age group: 37 cases treated over an 8-year period. Chang Gung Med J. 2010; 33(2): 152-156.

17. Frazier AL, Weldon C, Amatruda J. Fetal and neonatal germ cell tumors. Semin Fetal Neonatal Med. 2012; 17(4): 222-230.

Conflicto de intereses: Los autores declaran que no tienen. 